\title{
Ideación suicida, síntomas depresivos e involucramiento afectivo en adolescentes escolarizados del 2019
}

\author{
Sánchez, M., Maricarmen \\ Universidad de Panamá, Facultad de Psicología \\ Panamá, Panamá \\ maricarmen.sanchezm@outlook.com
}

\section{Abstract}

Introduction. Adolescence is a time of multiple physical, social and psychological changes, and there is a greater vulnerability to experience mental health conditions and risk behaviors that can lead to suicidal ideation. The aim of this study was to screen for the presence of suicidal ideation, depressive symptoms and affective involvement. Method. This was a descriptive-correlational non-experimental cross-sectional study. The sample consisted of 122 adolescents ages 15 to 18. Results. Suicidal ideation was reported by $30.3 \%$ of adolescents. From this group with suicidal ideation, $70.3 \%$ presented depressive symptomatology at moderate and severe levels. $51.4 \%$ presented functional affective involvement and $48.6 \%$ reported an absence; $45.6 \%$ presented dysfunctional involvement and $48.6 \%$ reported an absence. An average negative correlation was found between depression and functional affective involvement $(r=-0.48)$, and a low positive correlation between depression and dysfunctional affective involvement $(r=0.14)$. Conclusions. The adolescents reported the presence of suicidal ideation and high depressive symptoms, and it was also observed that many of these adolescents do not perceive within their family environment affective bonds that could play a protective role. It was observed how vital it is to inquire about the presence of suicidal ideation in school settings.

Keywords: suicidal ideation, depression, adolescent, affective involvement, suicidal behavior.

\section{Resumen}

Introducción. La adolescencia es una etapa donde se presentan múltiples cambios físicos, sociales y psicológicos, y se observa mayor vulnerabilidad a experimentar condiciones de salud mental y conductas de riesgo que pueden conducir a presentar ideación suicida. El objetivo de este estudio consistió en realizar un cribado sobre la presencia de ideación 
suicida, síntomas depresivos e involucramiento afectivo. Método. No experimentaltransversal de tipo descriptivo-correlacional. La muestra fue de 122 adolescentes con edades entre 15-18 años. Resultados. El 30.3\% de adolescentes reportó presencia de ideación suicida. De este grupo con ideación suicida el $70.3 \%$ presentó sintomatología depresiva en niveles moderados y graves. En cuanto al involucramiento afectivo funcional, el $51.4 \%$ lo presenta y el $48.6 \%$ reporta ausencia; el aspecto de involucramiento disfuncional, el $45.6 \%$ lo presenta y el $48.6 \%$ reporta ausencia. Se encontró una correlación negativa promedio entre depresión e involucramiento afectivo funcional $(r=-0.48)$, y una correlación positiva baja entre depresión e involucramiento afectivo disfuncional $(r=0.14)$. Conclusiones. Los adolescentes reportaron presencia de ideación suicida y síntomas depresivos altos, de igual manera se observa que mucho de estos adolescentes no perciben dentro de su entorno familiar vínculos afectivos que puedan cumplir un rol protector. Se observó lo vital que es indagar sobre la presencia de la ideación suicida en ámbitos escolares.

Palabras claves: ideación suicida, depresión, adolescente, involucramiento afectivo, conducta suicida.

\section{INTRODUCCIÓN}

El suicidio es un tema de salud pública a nivel mundial. Anualmente más de 800,000 personas mueren por suicidio, lo que sugiere que cada 40 segundos ocurre una muerte por suicidio y por cada uno que lo consigue aproximadamente 20 personas lo han intentado. En el 2016, el suicidio fue la segunda causa principal de defunción en el grupo etario de 15 a 29 años en todo el mundo [1]. En Panamá, en el año 2018 se registró que las lesiones autoinfligidas junto a los accidentes, agresiones y otra forma de violencia se encontraron dentro de las tres principales causas de muertes del país [2]. En un estudio sobre las características epidemiológicas del suicidio en Panamá entre el 2007 y 2016 se reportaron 1,475 muertes por suicidio de las cuales 184, es decir, el 13\% ocurrió en el grupo de 10 a 19 años [3]. Sin embargo, Panamá presenta un subregistro estimado del 14.4\% [3] lo que impide tener datos exactos [4]. La conducta suicida se entiende como un continuum amplio que abarca desde la ideación suicida, la amenaza o el gesto suicida, el intento suicida hasta el suicidio [1,5]. Esto permite entender el suicidio como un proceso que pasa por diversas etapas que hacen que se pueda prevenir y el mismo debe abordarse desde un enfoque multifactorial en el cual intervienen factores psicológicos, sociales, biológicos, culturales y ambientales [6]. La ideación suicida es considerada la primera fase de la conducta suicida $[7,8]$. Y es definida como los pensamientos, ideas, deseos e intenciones de servirse como 
agente de la propia muerte [9]. La presencia de ideas suicidas es una señal temprana de vulnerabilidad [10] y su presencia persistente puede significar un factor de riesgo que puede conducir a un acto suicida y que es resultado de diversos aspectos que apresuran o favorecen su aparición [11]. En el desarrollo de la adolescencia se puede presentar la ideación suicida como estrategia y respuesta frente a problemas/crisis que sobrepasan las capacidades de afrontamiento de los adolescentes. Sin embargo, existe un alto riesgo cuando el adolescente ve como única salida para la solución de sus problemas y sufrimiento terminar con su propia vida $[6,10,12]$. Muchos adolescentes no cuentan con las habilidades para manejar la frustración y resolver conflictos propios de la etapa y esto puede ser decisivo en la aparición y formación de ideación suicida [11]. La mitad de los trastornos mentales comienzan a los 14 años o antes, pero en su mayoría no se detectan ni se tratan [13]. En Panamá, aunque existen algunas investigaciones sobre el tema, ésta es escasa en población adolescentes. De ahí la importancia de realizar este estudio que establece como objetivo determinar la presencia de ideación suicida en los adolescentes tomando en cuenta que es un indicador de vulnerabilidad temprana y que en muchas ocasiones se deja pasar por alto y solo se llega a dar una intervención cuando los jóvenes se ven afectados por alguna condición de salud mental o han tenido un intento suicida. Al igual que observar la presencia de síntomas depresivo e involucramiento afectivo en los entornos familiares, estableciendo como hipótesis correlacional que a mayor involucramiento afectivo funcional menor es la presencia de síntomas depresivos. Considerando que la forma en que la familia mantiene síntomas depresivos, desesperanza e ideación suicida es a través de su funcionamiento [14].

\section{MÉTODO}

\section{A. Diseño del estudio}

No experimental transversal, no se manipuló variables y se recolectó la información en un solo momento y tiempo. El tipo de investigación es descriptivo-correlacional, se buscaba describir las variables estudiadas en los adolescentes escolarizados y la relación que existe entre síntomas depresivos e involucramiento afectivo en los adolescentes que presentaron ideación suicida.

\section{B. Población y muestra}

Unidad de análisis estuvo compuesta por adolescentes entre 15 a 18 años cursando estudios en cuarto y quinto año de un colegio público de la ciudad de Panamá y que estaban debidamente matriculados. El método empleado para la selección de la muestra fue no probabilístico de tipo voluntario donde se invitó a participar en el estudio a la mayor cantidad de estudiantes [15]. La muestra final estuvo conformada por 122 adolescentes, de 
los cuales 49 adolescentes eran masculinos y 73 adolescentes femeninas. Posteriormente se dividió la muestra en el grupo de adolescentes que presentó ideación suicida y los que reportaron ausencia de ideación suicida.

\section{Instrumentos y variables operacionales}

Los instrumentos que se utilizaron en este estudio fueron el Inventario de Depresión de Beck-II (BDI-II) para evaluar los niveles de síntomas depresivos y la presencia de ideación suicida con el ítem 9 del inventario [12] y el Cuestionario de Evaluación del Funcionamiento Familiar para el involucramiento afectivo funcional el punto de corte es de 51 puntos, a mayor valor se percibe mayor grado de involucramiento afectivo funcional y para el involucramiento afectivo disfuncional el punto de corte es de 33 puntos, a mayor valor se percibe mayor grado de involucramiento afectivo disfuncional [16].

\section{RESULTADOS}

\section{A. Tablas}

La Tabla 1 muestra la configuración de los grupos de adolescentes que reportaron presencia y ausencia de ideación suicida. Se observa que del total de la muestra de adolescentes escolarizados entre 15 a 18 años, el 30,3 \% (n: 37) presentó ideación suicida y el 69,7 \% (n: 85) manifestó ausencia de ideación suicida al momento de la evaluación.

Tabla 1. Porcentaje de adolescente con presencia y ausencia de ideación suicida, 2019-2020.

\begin{tabular}{lllll}
\hline \multirow{2}{*}{ Sexo } & \multicolumn{2}{l}{ Con ideación suicida } & \multicolumn{2}{l}{ Sin ideación suicida } \\
& $\mathrm{n}$ & $\%$ & $\mathrm{n}$ & $\%$ \\
\hline Femenino & 29 & 78,4 & 44 & 51,8 \\
Masculino & 8 & 21,6 & 41 & 48,2 \\
Total & 37 & $\mathbf{3 0 , 3}$ & $\mathbf{8 5}$ & $\mathbf{6 9 , 7}$ \\
\hline
\end{tabular}

En la Tabla 2 se observa que el grupo con ideación suicida presenta mayor porcentaje de sintomatología depresiva al momento de la evaluación en comparación con el grupo que reporta ausencia de ideación suicida.

Tabla 2. Porcentaje de síntomas depresivos en los adolescentes con y sin ideación suicida, 2019- 2020.

\begin{tabular}{lllll}
\hline & \multicolumn{2}{c}{ Con ideación suicida } & \multicolumn{2}{c}{ Sin ideación suicida } \\
Depresión & $\mathbf{n}$ & $\mathbf{\%}$ & $\mathbf{n}$ & $\%$ \\
\hline Síntomas altos & 26 & 70.3 & 11 & 12,9 \\
Síntomas bajos & 11 & 29.7 & 74 & 87,1 \\
Total & $\mathbf{3 7}$ & $\mathbf{1 0 0}$ & $\mathbf{8 5}$ & $\mathbf{1 0 0}$ \\
\hline
\end{tabular}


Según el involucramiento afectivo funcional (IAF) en la Tabla 3, al comparar los porcentajes entre los adolescentes con y sin ideación suicida se observó que el grupo sin ideación suicida reporta mayor percepción de involucramiento afectivo funcional en el entorno familiar. Sin embargo, en el grupo con ideación suicida los porcentajes no son tan diferentes, es decir, que cierto porcentaje percibe funcionalidad mientras que el otro porcentaje no lo percibe.

Tabla 3. Percepción del involucramiento afectivo funcional (IAF) en adolescentes con y sin ideación suicida, 2019-2020

\begin{tabular}{lllll}
\hline & \multicolumn{2}{c}{ Con ideación suicida } & \multicolumn{2}{c}{ Sin ideación suicida } \\
IAF & $\mathbf{n}$ & $\mathbf{\%}$ & $\mathbf{n}$ & $\mathbf{\%}$ \\
\hline Presencia de IAF & 19 & 51.4 & 75 & 88,2 \\
Neutro & 0 & 0.0 & 0 & 0,0 \\
Ausencia de IAF & 18 & 48.6 & 10 & 11,8 \\
Total & $\mathbf{3 7}$ & $\mathbf{1 0 0}$ & $\mathbf{8 5}$ & $\mathbf{1 0 0}$ \\
\hline
\end{tabular}

En cuanto a la percepción del involucramiento afectivo disfuncional (IAD) se observa en la Tabla 4 que hubo un mayor porcentaje de adolescentes sin ideación suicida que manifiesta ausencia de la percepción de involucramiento afectivo disfuncional. En contraste, en el grupo de adolescentes con ideación suicida los porcentajes son cercanos entre sí sobre ausencia y presencia de disfuncionalidad en su entorno en cuanto a la afectividad.

Tabla 4. Percepción del involucramiento afectivo disfuncional (IAD) en adolescentes con y sin ideación suicida, 2019-2020

\begin{tabular}{ccccc}
\hline & \multicolumn{2}{c}{$\begin{array}{c}\text { Con ideación suicida } \\
\text { \% }\end{array}$} & $\mathbf{n}$ & Sin ideación suicida \\
IAD & $\mathbf{n}$ & 45,9 & 23 & 27,1 \\
\hline Presencia de IAD & 17 & 5,4 & 6 & 7,1 \\
Neutro & 2 & 48,6 & 56 & 65,9 \\
Ausencia de IAD & 18 & 100 & 85 & 100 \\
Total & 37 & & &
\end{tabular}

En cuanto a el análisis correlacional entre los síntomas depresivos e involucramiento afectivo funcional en el grupo con ideación suicida se obtuvo una correlación negativa promedio significativa ( $r=-0.48 ; p=0.002)$, lo que sugiere que a mayor involucramiento afectivo funcional en el entorno familiar menor sintomatología depresiva en la muestra evaluada. En contraste, la correlación entre los síntomas depresivos e involucramiento afectivo disfuncional en el grupo con ideación suicida fue positiva baja, no significativa ( $r$ = $0.14, p=0.40)$. 


\section{CONCLUSIONES}

Nuestro estudio mostró que los adolescentes reportan ideación suicida, síntomas depresivos y dificultades en el funcionamiento familiar en el aspecto afectivo. Con estos datos resaltamos que los colegios tienen un papel fundamental en la detección y prevención de problemas relacionados a la salud mental y conductas de riesgo como el suicidio. $Y$ reconocemos que en nuestra sociedad panameña son necesarias las políticas públicas y leyes en temas de salud mental que brinden atención temprana y con enfoques preventivos. El trabajo multidisciplinario en ámbitos educativos, atención a la salud y la comunidad es fundamental para la prevención, fortaleciendo los factores protectores y disminuyendo los factores de riesgo, al mismo tiempo contribuye a eliminar el estigma para que las personas busquen ayuda y se reduzcan las conductas de riesgo y sobre todo muertes por suicidio.

\section{Referencias}

[1] Organización Mundial de la Salud (OMS). Prevención del suicidio: un imperativo global. Washington, DC: OPS, 2014.

[2] Instituto Nacional de Estadística y Censo (INEC). Estadísticas Vitales, Volumen III Defunciones: 2018. Ciudad de Panamá, Panamá, 2020. Disponible en: https://www.inec.gob.pa/archivos/ P0579518620191205123511Cuadro\%2025.pdf

[3] Núñez-Samudio V, Jiménez-Domínguez A, López Castillo H, Landires I. Epidemiologic Characteristics of Suicide in Panama, 2007-2016. Medicina [Internet]. 2020; 56(9):442. Disponible en: https://doi. org/10.3390/medicina56090442

[4] Saéz-Llorens, X. Suicidio en adolescentes: factores precipitantes y guías de prevención. In: Salud mental y suicidio: Rompamos el silencio: V. Maduro, M. Mayo, X. Saéz, P. Vargas, Á. Gómez, M. Plata y F. Mizrachi. Panamá: Nomos S.A; 2018. p. 47- 53.

[5] Almonte, C. Espectro suicida en la infancia y adolescencia. In: Psicopatología infantil y de la adolescencia. 2 ed. Manual Modern, 2012. p. 560-568.

[6] Mayo, M. Suicidio, un problema de salud pública. In: Salud mental y suicidio: Rompamos el silencio: V. Maduro, M. Mayo, X. Saéz, P. Vargas, Á. Gómez, M. Plata, C. Sosa, F. Mizrachi Panamá: Nomos S.A; 2018. p.15-20.

[7] De la Torre, M. Protocolo para la detección y manejo inicial de la ideación suicida. Universidad Autónoma de Madrid[internet]. 2013 [citado 20 de noviembre 2020].

[8] Sánchez, J., Villarreal, M. E., Musitu, G., Martinez, F. Ideación Suicida en Adolescentes: Un Análisis Psicosocial. Psychosocial Intervention. [internet]. 2020; 19(3), 279-287. Disponible en: http://scielo. isciii.es/scielo.php?script=sci arttext\&pid=S1132-05592010000300008

[9] Toro, R., Grajales, F., \& Sarmiento, C. Riesgo suicida según la tríada cognitiva negativa ideación, desesperanza y depresión. Aquichan [Internet]. 2016; 16(4), 473-486. Disponible en: http://www. scielo.org.co/scielo.php?script=sci_abstract\&pid=S1657- 59972016000400473\&lng=es

[10] . Gómez, A. Evaluación del riesgo de suicidio: enfoque actualizado. Revista Médica Clínica Las Condes [internet]. 2012; 23(5), 607-615. Disponible en: https://www.sciencedirect.com/science/ article/pii/S0716864012703553

[11] Ávila, DS. Nivel de prevalencia de ideas e intentos suicidas en los estudiantes de sexto semestre de 
seis preparatorias de montemorelos [internet]. 2014 [citado 20 de noviembre de 2020]. Disponible en: http://dspace.biblioteca.um.edu.mx/xmlui/handle/20.500.11972/641

[12] Guevara-Werlang, Esteves, M. Identificación de Potencial Suicida en Adolescentes de 13 a 19 Años. Revista Argentina de Clínica Psicológica [internet].2012; XXI (3), 281-289. Disponible en: https:// dialnet.unirioja.es/servlet/articulo?codigo $=4258302$

[13] Organización Mundial de la Salud (OMS) [internet]. Salud mental del adolescente. [citado 20 de noviembre del 2020]. Disponible en: https://www.who.int/es/news-room/fact sheets/detail/ adolescent-mental-health

[14] Eguiluz, L., Ayala, M. Relación entre ideación suicida, depresión y funcionamiento familiar en adolescentes. Psicología Iberoamericana [internet] 2014; 22(2), 72-80. Disponible en: http:// revistas.ibero.mx/psicologia/articulo detalle.php?id volumen=11\&id articulo=124

[15] Hernández S. Metodología de la investigación. 6ta edición. México D.F: McGraw-Hill, 2014: p. 386387

[16] Atri, R., Zetune. Cuestionario de Evaluación del Funcionamiento Familiar (EFF) In Instrumentos de evaluación en terapia familiar y de pareja: M. L. Velasco, M. D. Luna México, D.F: Pax México; 2015. p. 1-27.

\section{Autorización y Licencia CC}

Los autores autorizan a APANAC XVIII a publicar el artículo en las actas de la conferencia en Acceso Abierto (Open Access) en diversos formatos digitales (PDF, HTML, EPUB) e integrarlos en diversas plataformas online como repositorios y bases de datos bajo la licencia CC:

Attribution-NonCommercial-ShareAlike 4.0 International (CC BY-NC-SA 4.0) https://creativecommons.org/ licenses/by-nc-sa/4.0/.

Ni APANAC XVIII ni los editores son responsables ni del contenido ni de las implicaciones de lo expresado en el artículo. 